Revista Internacional de Ciencias Podológicas

ISSN: 1989-5151

\title{
Efectividad de la terapia de presión negativa en la cura de úlceras de pie diabético: revisión sistemática
}

González-Ruiz, M. ${ }^{1}$; Torres-González, J. I.²; Pérez-Granda, M. J.'; Leñero-Cirujano, M. ${ }^{4}$ Corpa-García, A. ${ }^{5}$; Jurado-Manso, J.6; Gómez-Higuera, J. ${ }^{7}$

Fecha de recepción: 7 enero de 2017 / Fecha de aceptación: 22 de abril de 2017

\section{Resumen.}

Introducción: Las úlceras de pie diabético son una de las mayores causas de incapacidad en pacientes con Diabetes Mellitus (DM). La Terapia de Presión Negativa (TPN) se presenta como uno de los mejores procedimientos en su tratamiento, estimulando la granulación, drenando el exudado y reduciendo el edema.

Objetivos: Realizar una revisión sistemática acerca de la efectividad y la aplicabilidad clínica real del uso de la TPN en las úlceras de pie diabético.

Material y métodos: Búsqueda bibliográfica realizada en noviembre de 2016 en las bases de datos PubMed, CINAHL, SciELO y Web of Science, con las palabras clave ("negative pressure wound therapy" OR "vacuum assisted closure" OR "vacuum sealing drainage") AND ("diabetic foot" OR "diabetic wound" OR "diabetic ulcer"). Se seleccionaron las publicaciones cuyos diseños fueran ensayos clínicos aleatorizados (ECA), según los criterios de la declaración PRISMA, y analizaran el objetivo planteado.

Resultados: Se encontraron 12 ECAs de calidad metodológica suficiente. 7 de los ECAs utilizaron como variable principal la superficie del lecho de la herida y 5 utilizaron el tiempo hasta la granulación.

Conclusiones: La TPN consigue una mayor superficie de tejido de granulación, un menor tiempo de cicatrización y una diminución del tiempo hasta que comienza la granulación.

Palabras clave: pie diabético; Terapia de Presión Negativa.

\section{[en] Effectiveness of negative pressure therapy in diabetic foot ulcer: systematic review}

\begin{abstract}
.
Background: Diabetic foot ulcers are one of the major causes of disability in patients with Diabetes Mellitus (DM). The Negative Pressure Wound Therapy (NPWT) is one of the major procedures in his treatment, estimulating granulation, draining exudate and reducing edema.e

Objectives: To realize a systematic review about the effectiveness and the real clinical application of NPWT use in diabetic foot ulcers.
\end{abstract}

1 González-Ruiz M à RN; MsC. Colaborador Departamento de Enfermería. Facultad de Enfermería, Fisioterapia y Podología. Universidad Complutense de Madrid.marcgonz@ucm.es

2 Torres-González JI à RN; MsC; PhD. Oncología Radioterápica. Hospital Clínico San Carlos. Instituto de Investigación Sanitaria San Carlos (IdISSC). Profesor Asociado. Departamento de Enfermería. Facultad de Enfermería, Fisioterapia y Podología. Universidad Complutense de Madrid. jitorres@ucm.es

3 Pérez-Granda MJ à RN; MsC; PhD. Departamento de Anestesiología. Hospital General Universitario Gregorio Marañón. Ciber de Enfermedades Respiratorias (CIBERES) CIBER (CB06/06/0058). Departamento de Microbiología Clínica y Enfermedades Infecciosas. Instituto de Investigación Sanitaria Gregorio Marañón (IiSGM). Profesora Asociada de Ciencias de la Salud. Departamento de Enfermería. Facultad de Enfermería, Fisioterapia y Podología. Universidad Complutense de Madrid. massus@ hotmail.es

4 Leñero-Cirujano M à RN; MsC. Colaborador Departamento de Enfermería. Facultad de Enfermería, Fisioterapia y Podología. Universidad Complutense de Madrid.mlcirujano@ucm.es

5 Corpa-García A à RN. Colaborador Departamento de Enfermería. Facultad de Enfermería, Fisioterapia y Podología. Universidad Complutense de Madrid. alecorpa@ucm.es

6 Jurado-Manso J à RN. Colaborador Departamento de Enfermería. Facultad de Enfermería, Fisioterapia y Podología. Universidad Complutense de Madrid. jjurad01@ucm.es

7 Gómez-Higuera J à RN; MsC; PhD. Profesor Titular. Departamento de Enfermería. Facultad de Enfermería, Fisioterapia y Podología. Universidad Complutense de Madrid. jacintogomez@enf.ucm.es 
Methods: The literature search has been made in November 2016 in PubMed, CINAHL, SciELO and Web of Science databases, with the kew words ("negative pressure wound therapy" OR "vacuum assisted closure" OR "vacuum sealing drainage") AND (“diabetic foot" OR "diabetic wound" OR “diabetic ulcer"). Were selected publications whose designs were randomized clinical trials (RCTs), according PRISMA declaration criteria, and whose objective was to analyze the study objective.

Results: Twelve RCTs were found with sufficient methodological quality. 7 of the RCTs used as the main variable the surface of the wound bed and 5 used the time to granulation.

Conclusions: NPWT get a greater granulation area, a smaller wound healing time and a minor time to start the granulation.

Key words: diabetic foot; Negative-Pressure Wound Therapy.

Los autores declaran no tener ningún tipo de interés económico o comercial.

Sumario. 1. Introducción. 2. Material y métodos. 2.1. Métodos de búsqueda. 2.2 Criterios de inclusión. 2.3. Criterios de exclusión. 2.4. Selección y análisis de la calidad de los estudios. 3. Resultados. 3.1 Búsqueda y selección de los artículos. 3.2. Superficie y lecho de la herida. 3.3. Tiempo hasta la granulación. 4. Discusión.. 5. Conclusiones. 6. conflicto de intereses. 7. Bibliografía.

Cómo citar: González-Ruiz, M.; Torres-González, J. I.; Pérez-Granda, M. J.; Leñero-Cirujano, M.; Corpa-García, A.; Jurado-Manso, J.; Gómez-Higuera J. (2018) Efectividad de la terapia de presión negativa en la cura de úlceras de pie diabético: revisión sistemática, en Revista internacional de ciencias podológicas 12(1), 1-13

\section{Introducción}

La Diabetes Mellitus (DM) es uno de los principales problemas de salud que se encuentran actualmente en la sociedad, llegando a ser considerada pandemia por la Organización Mundial de la Salud (OMS) ${ }^{1}$. Numerosos estudios epidemiológicos datan que el número de personas afectadas por DM ha aumentado exponencialmente en las últimas dos décadas: desde los 108 millones de casos en todo el mundo en la década de los 80 , hasta los 422 millones de personas afectadas en 2014. Además, se estima que en 2030 el número de personas con DM ascenderá a más de 360 millones en todo el mundo ${ }^{1,2}$.

Los pacientes que sufren DM están sujetos a multitud de complicaciones, dentro de las cuales destacan las úlceras, un problema bastante común entre las personas diabéticas $\mathrm{y}$ que se ha visto incrementado desde hace varias décadas hasta hoy en día ${ }^{1,3}$. Aunque parece ser que los factores más determinantes para presentar estas úlceras son la enfermedad vascular y la neuropatía periférica, son muchos los factores que predisponen a padecerlas: una larga duración del proceso de enfermedad, la edad avanzada, un pobre control de la glucemia, el uso de calzado inadecuado, los hábitos tabáquicos, un nivel socioeconómico bajo o factores psicológicos ${ }^{2,3}$. Actualmente, se estima que el $15 \%$ de todos los pacientes con DM han tenido o tendrán úlceras diabéticas en algún momento de su vida, lo cual evidencia la amplia magnitud del problema ${ }^{2}$.

En general, las complicaciones más frecuentes de estas úlceras son infección, osteomielitis y celulitis ${ }^{1,2}$, pudiendo derivar en la amputación del miembro afectado con un mal cuidado de las mismas. La amputación es una medida muy agresiva que se asocia a consecuencias sociales y psicológicas muy graves, con aumento de la morbimortalidad en el paciente y un impacto socioeconómico ${ }^{2,4}$.

Existen multitud de procedimientos en el cuidado de estas úlceras, sin embargo, en los últimos años está adquiriendo un papel clave la cura de úlceras con Terapia de Presión Negativa (TPN). La TPN consiste en un sistema no invasivo que utiliza una presión negativa controlada mediante un dispositivo conectado al vacío que promueve la estimulación y la curación de la herida ${ }^{4,5}$. Los beneficios de la TPN se obtienen fundamentalmente debido a dos principios o mecanismos básicos: el control del exudado y la estimulación local celular de la herida. Entre las ventajas de este sistema destaca el aumento del flujo local sanguíneo, la estimulación del proceso de granulación y la angiogénesis, el 
drenado del exudado y la reducción del edema y colonización bacteriana ${ }^{5,6}$.

No obstante, a pesar de la evidencia existente en torno al uso de la TPN en la cura de heridas quirúrgicas, vasculares o de cualquier otro tipo $^{7-10}$, son escasos los estudios que analicen su eficacia en las úlceras diabéticas.

Por todo ello, el objetivo de este estudio es realizar una revisión sistemática de todos los artículos publicados hasta la fecha para analizar la efectividad y la aplicabilidad clínica real del uso de la TPN en las úlceras de pie diabético.

\section{Material y métodos}

\subsection{Método de búsqueda}

Se realizó una búsqueda bibliográfica en las bases de datos PubMed, CINAHL, SciELO y Web of Science en todos los idiomas, en un rango de fechas de 2000-2016, utilizando los siguientes operadores booleanos: ("negative pressure wound therapy" OR "vacuum assisted closure" OR "vacuum sealing drainage") AND ("diabetic foot" OR "diabetic wound" OR "diabetic ulcer").

\subsection{Criterios de inclusión}

Los criterios de inclusión aplicados en las bases de datos anteriormente mencionadas fueron los siguientes:

- Artículos originales científicos publicados en el rango de años 2000-2016.

- Artículos escritos en cualquier idioma.

- Estudios cuyos diseños fueran Ensayos Clínicos Aleatorizados (ECA) o cuasiexperimentales.

- Estudios cuya muestra de población fuera el individuo adulto, con edades comprendidas entre los 18 y los 65 años.

- Estudios cuyos objetivos fueran evaluar la efectividad y/o eficacia de la TPN en la curación de las úlceras de pie diabético.

\subsection{Criterios de exclusión}

Los criterios de exclusión aplicados en las bases de datos anteriormente mencionadas fueron los siguientes:
- Estudios cuyos objetivos fueran evaluar la efectividad y/o eficacia de la TPN en heridas y lesiones distintas a las producidas en el pie diabético.

- Artículos cuyos diseños fueran estudios observacionales o aquellos que fueran revisiones sistemáticas y/o metaanálisis.

- Protocolos de estudios no desarrollados aún o estudios duplicados.

- Estudios que aportaran información insuficiente para juzgar su calidad metodológica o la calidad de sus resultados.

\subsection{Selección y análisis de la calidad de los estudios}

Para la realización de la revisión, se siguieron los criterios indicados en la declaración PRISMA acerca de la elaboración de revisiones sistemáticas. Se realizaron sucesivos cribados realizados por dos revisores para seleccionar los artículos incluidos en la revisión: se excluyeron los artículos duplicados, aquéllos que no cumplían los criterios de inclusión tras la lectura del título y el resumen, aquéllos incompatibles con esta revisión tras un análisis del estudio completo y aquéllos con una calidad metodológica insuficiente.

Se evaluó la calidad metodológica de los estudios, considerando la existencia o no de errores sistemáticos debido al diseño del estudio y su realización (sesgos) y la probabilidad de errores aleatorios (intervalo de confianza de los resultados) para establecer la mayor precisión, validez interna y validez externa en la interpretación de los resultados.

Para ello, se utilizó la escala Jadad. Se seleccionaron aquellos estudios con una puntuación $\geq 3$, otorgándose a cada uno un nivel de calidad en función de la puntuación obtenida ( $3=$ calidad baja, $4=$ calidad moderada, $5=$ calidad alta).

Una vez seleccionados los artículos que se incluyeron finalmente en la revisión, los resultados se organizaron y representaron en una tabla, conteniendo la siguiente información referente a cada estudio:

- Objetivo del estudio.

- Diseño del estudio.

- Características de la muestra.

- Descripción breve de la intervención. 
- Resultados y conclusiones del estudio.

- Nivel de calidad del estudio.

Tras el análisis de los resultados encontrados en el proceso de búsqueda, los estudios analizados en la revisión fueron organizados en dos niveles, en función de la variable principal: superficie del lecho de la herida y tiempo hasta la granulación.

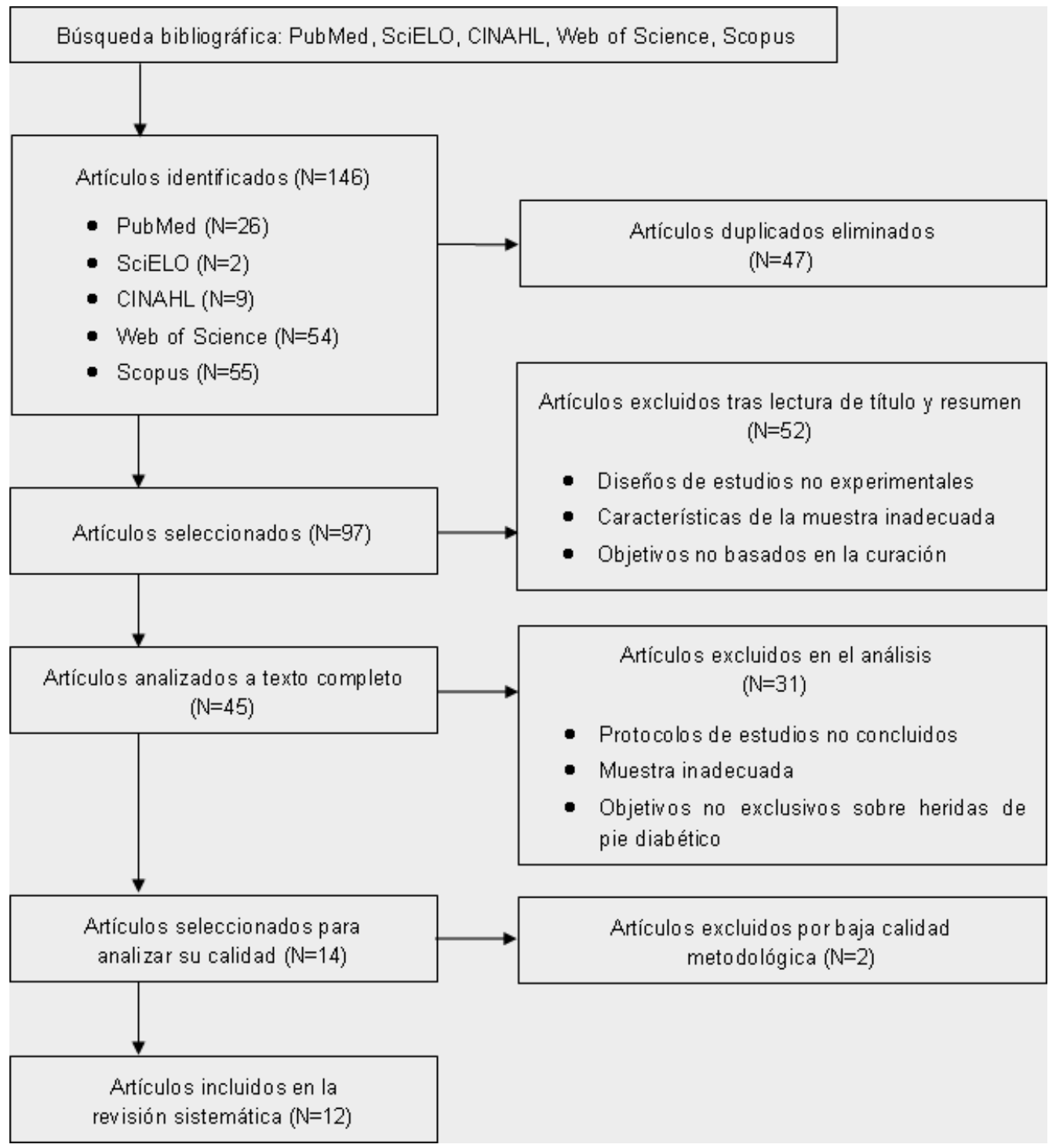

Figura 1. Diagrama de flujo de búsqueda y selección de los artículos

\section{Resultados}

\subsection{Búsqueda y selección de los artículos}

La figura 1 representa el proceso mediante el cual se seleccionaron los artículos incluidos finalmente en la revisión, aplicando el diagrama de flujo de la declaración PRISMA de revisiones sistemáticas.
Tras los procesos de cribado descritos en el esquema, se incluyeron 12 estudios en la revisión final: siete en el grupo de superficie del lecho de la herida y los cinco restantes en el subgrupo de tiempo hasta la granulación. Únicamente tres de los artículos poseían una calidad metodológica baja; el resto, con una puntuación de 4 en la escala de Jadad, tenían un nivel de calidad moderado.

En cuanto al diseño de los estudios, cabe destacar que los 12 artículos seleccionados 
tenían un diseño simple ciego, debido a la dificultad de efectuar un estudio doble ciego en el ámbito de la cura de heridas, donde a simple vista se puede evidenciar el método usado con cada sujeto.

\subsection{Superficie del lecho de la herida}

La variable principal que utilizaron los 7 ECAs $^{11-17}$, fue la superficie del lecho de la herida, para lo cual se midió la superficie con tejido de granulación o la cicatrización parcial y/o total (Tabla 1).

En la mayoría de los estudios ${ }^{11-16}$ se comparó la efectividad de la TPN frente a la cura convencional húmeda, posterior a una limpieza del lecho con solución salina y un proceso de desbridamiento en aquellas heridas que lo requirieran.

Por el contrario, el estudio de Lavery et $\mathrm{al}^{17}$ comparó heridas tratadas todas ellas con TPN, con la diferencia del tipo de material utilizado y la presión de vacío a la que se sometió la herida.

Únicamente el estudio de Blume et $\mathrm{al}^{16}$, analizó la TPN también en términos de seguridad, junto a la efectividad del propio proceso.

\subsection{Tiempo hasta la granulación}

El tiempo hasta la granulación se utilizó como variable principal de medida por cinco ECAs, midiendo el tiempo hasta el inicio de la granulación o la curación completa de la herida (Tabla 2).

La mayoría de los estudios ${ }^{18-22}$, analizó la eficacia de la TPN en términos de tiempo frente a la cura húmeda convencional, a excepción del estudio de Yang et $\mathrm{al}^{21}$, más orientado al componente fisiológico provocado en el lecho de la herida tras el uso de la TPN. Junto a éste, Karatepe et $\mathrm{al}^{20}$ abordó la efectividad de la TPN en términos de confort y calidad de vida, diferenciándola de la cura húmeda tradicional.

Sólo uno de los estudios, el realizado por Apelqvist et $\mathrm{al}^{22}$, abordó el estudio en términos de coste-efectividad, evaluando tanto los costes directos del uso de la TPN como los costes indirectos de visitas clínicas y procedimientos sanitarios. 


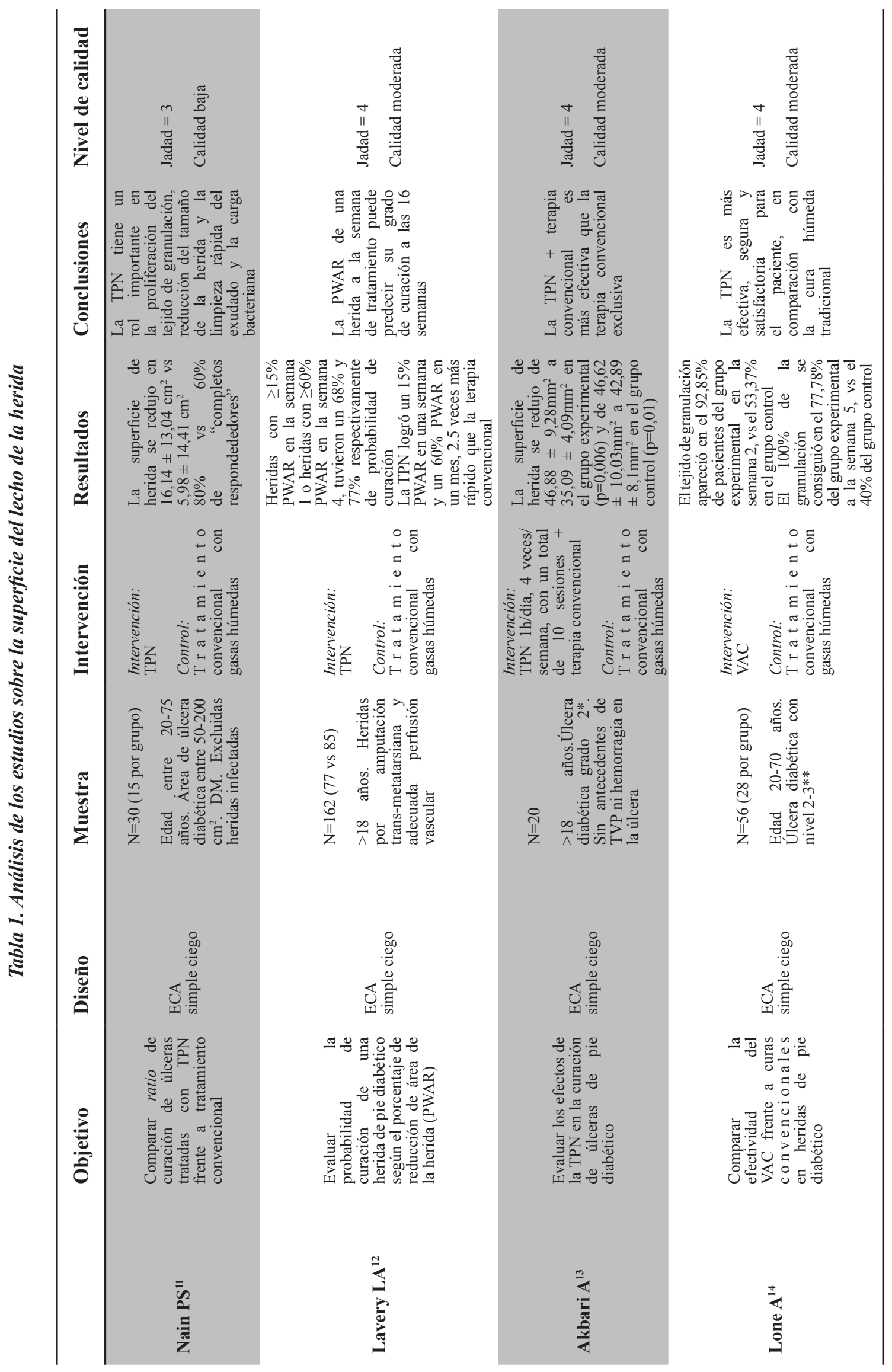




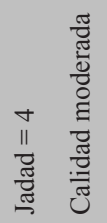

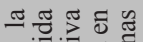

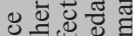

조요요

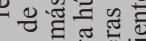

乙:

एक

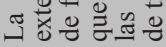

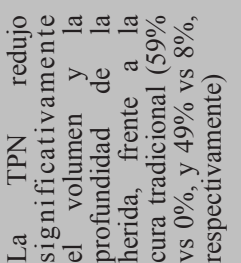

$\circ$ ठ요

$\Rightarrow>$

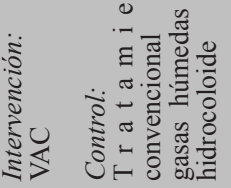

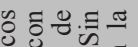

.

ज़ छ :

$\dot{0} \bar{v} \cdot \frac{0}{3}$

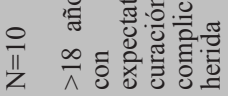

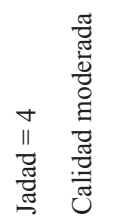

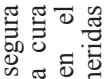

(⿻)丨

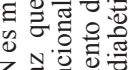

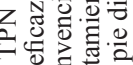

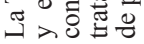

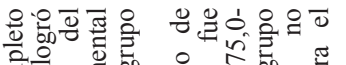

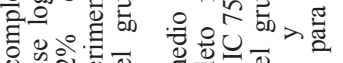

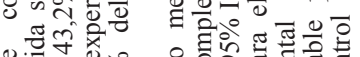

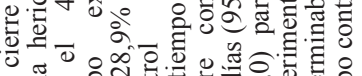

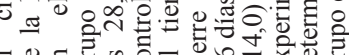
垗
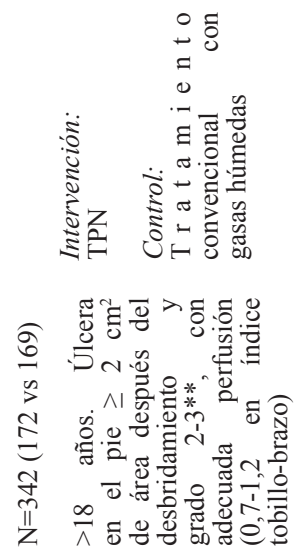

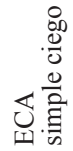

퓽 욥 $\bar{g}$

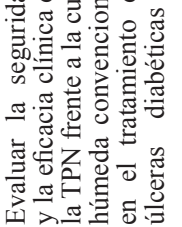

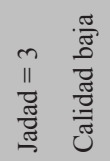

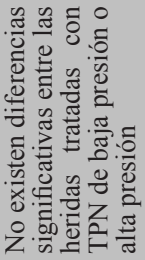

홍

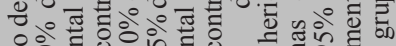

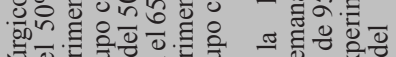

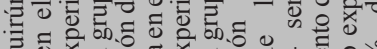

б

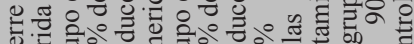

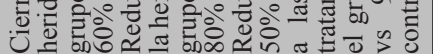
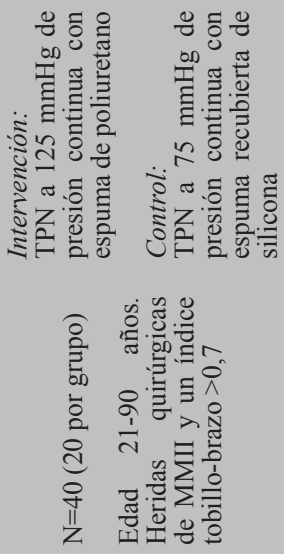


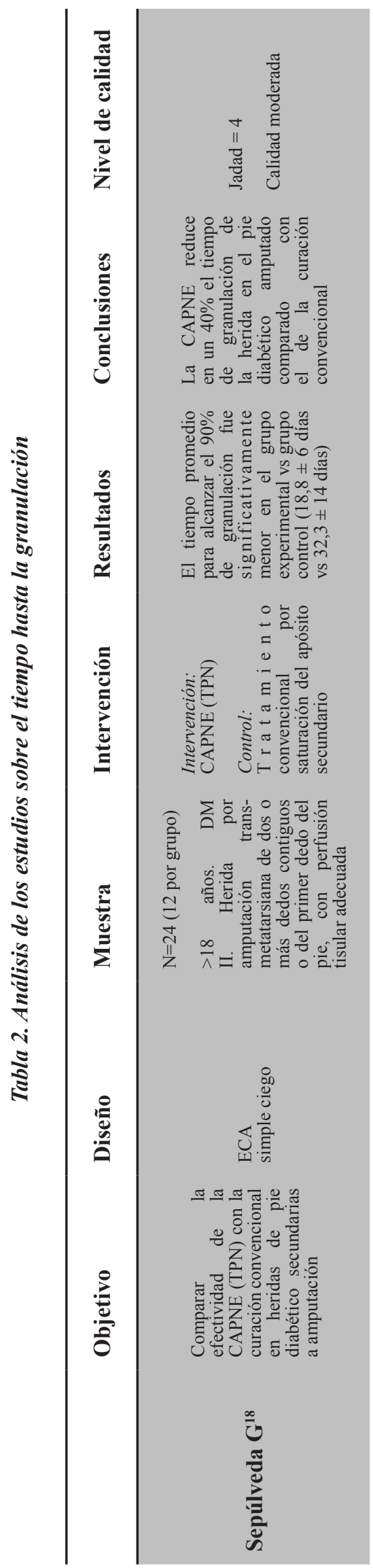

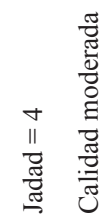

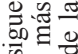

空。

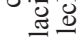

乙震要

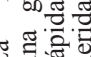

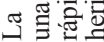

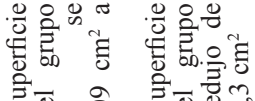

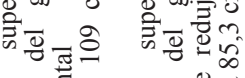

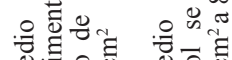

궁.융

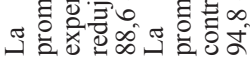

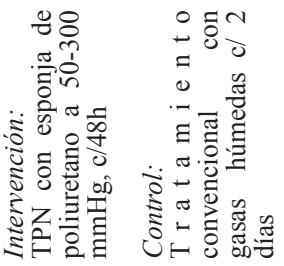

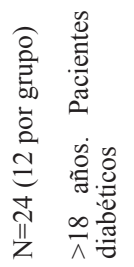

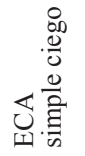

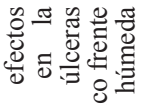

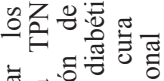

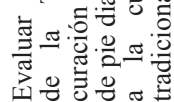

$\frac{1}{15}$

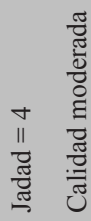

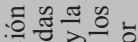

政

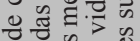

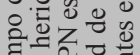

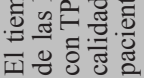

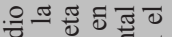

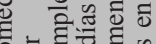

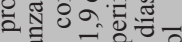

$\circ \frac{0}{\omega} \cdot \overline{0}$

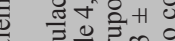

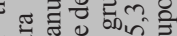

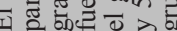

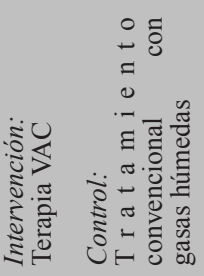

$\cong$

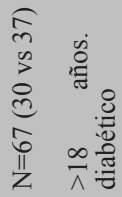

$$
\text { 它竞 }
$$

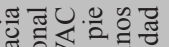

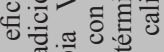

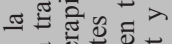

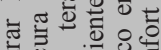

:

ठำ 


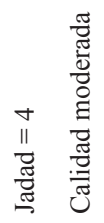

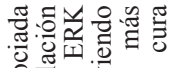

施드

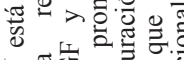

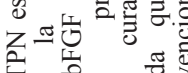

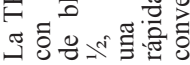

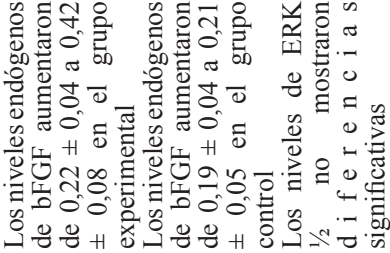

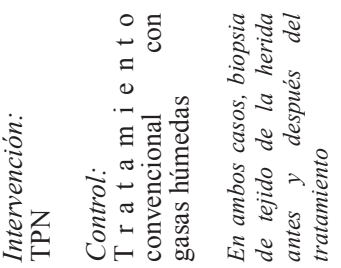

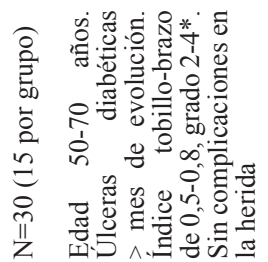
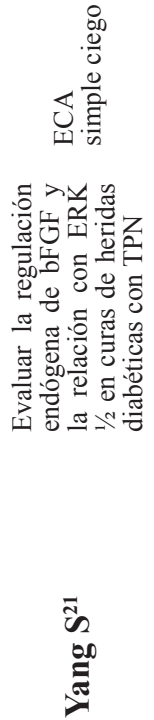
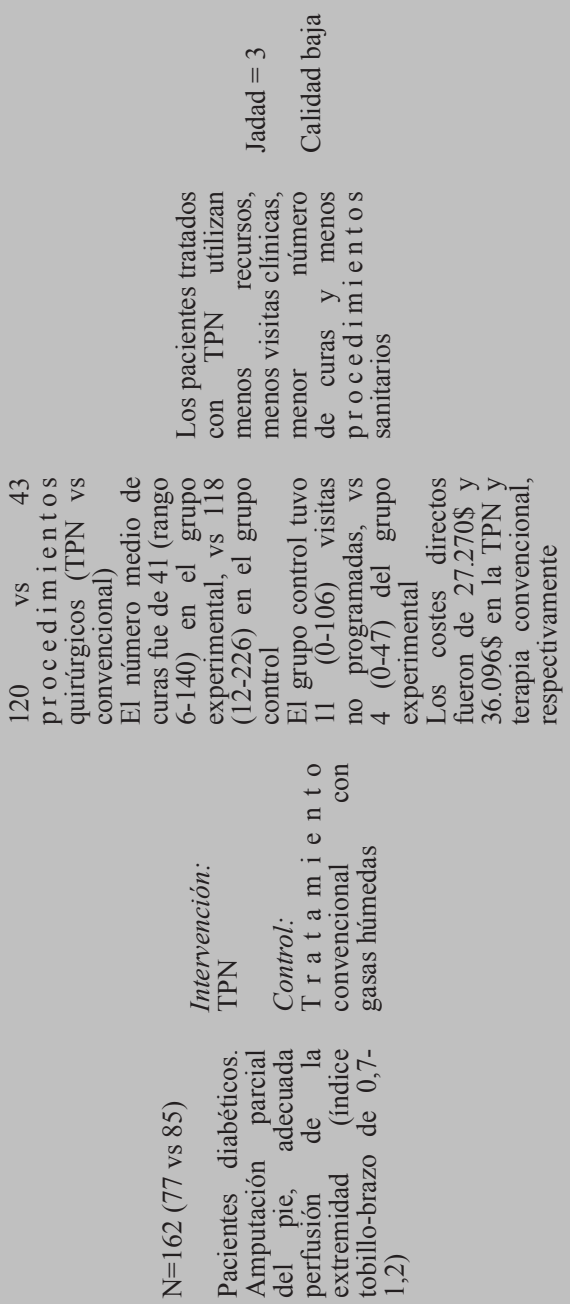

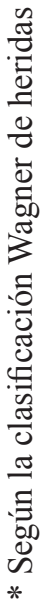




\section{Discusión}

Esta revisión sistemática parece demostrar una mayor efectividad de la TPN frente a la cura húmeda tradicional en el tratamiento de las úlceras de pie diabético. Concretamente, los datos sugieren que la TPN promueve una mayor superficie de tejido de granulación y reepitelización, disminuye la extensión de la herida y reduce el tiempo de curación (Tabla 1 y 2).

Se seleccionaron 20 ECAs, generalmente considerados como el estándar de calidad en la investigación de intervenciones, los cuales se consideraron que poseían la calidad metodológica necesaria para tener en cuenta su validez. Cabe destacar que se encontró un protocolo de ensayo clínico en fase de recogida de datos y que no pudo ser incluido en esta revisión, por lo que se recomienda incluirlo en revisiones posteriores una vez publicados sus resultados ${ }^{23}$.

Esta revisión pretende proporcionar una imagen completa y amplia acerca de la utilidad real de la TPN en la cura de heridas diabéticas. Sin embargo, resulta complejo valorar la validez real de la aplicabilidad clínica a partir de esta revisión, puesto que la mayoría de artículos seleccionados presentaron una muestra de pacientes muy pequeña con criterios de inclusión poco definidos. Por ello, creemos necesario añadir en futuros ECAs criterios de inclusión más severos, con control de factores de confusión tales como la edad, el sexo, la raza o el tipo y/o severidad de la diabetes. A pesar de ello, los datos encontrados parecen indicar que la TPN se encuentra asociada con un menor número de eventos adversos $\mathrm{y}$ complicaciones en la herida ${ }^{11,14,16}$.

Los estudios que utilizaron como variable principal la superficie del lecho de la herida concluyeron de manera similar: las heridas curadas con TPN obtuvieron mayor superficie de tejido de granulación en el tiempo de estudio y cicatrizaron más rápido que las curadas con gasas húmedas (Tabla 1). Estos resultados parecen sugerir una alta recomendación de usar TPN en heridas de pie diabético. Sin embargo, todos los estudios se realizaron en heridas crónicas con lechos limpios; por lo que se pone de manifiesto la necesidad de plantear en futuras investigaciones sujetos con heridas diabéticas de otras características, como por ejemplo la combinación de antibioterapia sistémica y TPN en heridas que presenten infección.

Los resultados de los estudios referentes al tiempo hasta la granulación sugieren que las heridas curadas con TPN granulan aproximadamente en la mitad de tiempo (Tabla 2). Esto puede ser debido fundamentalmente al propio mecanismo de acción de la TPN, que estimula la angiogénesis y la regeneración celular $^{24}$.

Aunque el objetivo de esta revisión es analizar la efectividad de la TPN en las heridas de pie diabético, resulta evidente el factor crucial que desempeñan los costes económicos en la elección de una técnica de cura u otra. Existen estudios que evalúan la relación coste-efectividad ${ }^{25-27}$, concluyendo, al igual que el analizado de Apelqvist et $\mathrm{al}^{22}$, la gran diferencia económica existente entre la TPN y cualquier otro tipo de cura.

Además de los costes directos, el menor número de días necesarios en la curación total de la herida, junto al menor número de cambio de apósito diario, disminuye notablemente el tiempo de hospitalización $\mathrm{y}$ de visitas ambulatorias sucesivas, tal y como analizó Trueman $\mathrm{P}$ en una población de Estados Unidos ${ }^{28}$. Este aspecto concuerda con un enfoque coste-efectividad, pues todo tratamiento breve generalmente resulta viable desde un punto de vista puramente económico.

Junto a los costes económicos, la literatura científica menciona los riesgos y las complicaciones potenciales derivados del uso de la TPN, como por ejemplo sangrado, irritación de la piel perilesional o infección de la herida ${ }^{4}$. Resultaría interesante abordar en futuros estudios la eficacia de la TPN en términos de seguridad, pues son pocos los ECAs de esta revisión que la utilizan como variable de estudio ${ }^{14,16}$. Además, junto a la seguridad del procedimiento, sería interesante analizar en futuras investigaciones el número de recidivas de heridas según un tratamiento u otro.

También se deben tener en cuenta los diferentes dispositivos de TPN que existen en el mercado, de diferentes marcas comerciales y materiales. Entre ellos, en los últimos años ha destacado el uso del Spiracur SNaP Wound Care System, ultraligero y portátil, más barato que otros dispositivos, aunque con la misma eficacia demostrada ${ }^{29,30}$.

Esta revisión trata de ser una de las primeras en abordar exclusivamente la efectividad de la 
TPN en la cura de heridas de pie diabético. Existen multitud de estudios, revisiones y metaanálisis sobre la eficacia y efectividad del uso de esta terapia en grandes quemados, heridas traumáticas o heridas quirúrgicas abiertas $^{30-36}$; sin embargo, a pesar de su gran prevalencia, resulta sorprendente el escaso número de revisiones y metaanálisis cuyos objetivos sean las heridas de pie diabético $4,37,38$.

Por último, dado que la mayor parte de la evidencia revisada se basa en pacientes con el lecho de la herida limpio, sin complicaciones asociadas, resultaría esencial realizar ensayos en poblaciones con heridas complejas, tales como infección o sobresangrado, para analizar las posibles diferencias existentes entre la aplicabilidad clínica de las intervenciones en un grupo de pacientes u otro.

En cuanto a las limitaciones del estudio, se encontraron las siguientes:

- Existen pocos ensayos clínicos aleatorizados sobre la utilidad clínica de la TPN en las heridas de pie diabético.

- Muchos de los estudios acerca de la efectividad de la TPN se realizan con muestras muy pequeñas, lo que puede limitar la generalización de sus conclusiones.

- Existe muy poca evidencia acerca de las diferencias de efectividad de la TPN en función de las complicaciones presentes en la herida.
- Los beneficios y los daños de las intervenciones deben ser evaluados de forma independiente antes de ponerlas en práctica.

- La terminología y nomenclatura utilizadas en los estudios resulta variada y poco clarificadora, dificultando el proceso de búsqueda.

\section{Conclusiones}

Las heridas de pie diabético constituyen uno de los principales problemas de salud pública en la actualidad cuya curación con TPN es posible, a tenor de los resultados obtenidos en esta revisión.

Esta revisión ha evidenciado un alentador auge de la literatura científica acerca de la efectividad del uso de TPN en heridas diabéticas. Los resultados parecen indicar que la TPN consigue una mayor superficie de tejido de granulación, con la consiguiente mayor rapidez de cicatrización.

Aun así, resulta necesario realizar futuros estudios que evalúen el impacto que la TPN tiene en el tratamiento de las úlceras de pie diabético, con el objetivo de incorporarla a la práctica clínica diaria.

\section{Conflicto De Intereses}

Los autores declaran no tener ningún conflicto de intereses.

\section{Bibliografía}

1. Organización Mundial de la Salud (OMS). Informe Mundial sobre la Diabetes [Internet]. Informe Mundial sobre la Diabetes. 2016. Disponible en: http://www.idf.org/node/26452?language=es.

2. Asociación Española de Enfermería Vascular y Heridas. Guía de práctica clínica: Consenso sobre úlceras vasculares y pie diabético. Segunda edición. Sevilla: AEEVH, 2014.

3. Mokdad AH, Ford ES, Bowman BA, Dietz WH, Vinicor F, Bales VS, et al. Email Alerts Prevalence of Obesity, Diabetes, and Obesity-Related Health Risk Factors. Jama. 2003;289(1):76-9.

4. Isaac AL, Armstrong DG. Negative pressure wound therapy and other new therapies for diabetic foot ulceration: The current state of play. Med Clin North Am [Internet]. 2013;97(5):899-909. Disponible en: http://dx.doi.org/10.1016/j.mcna.2013.03.015.

5. Vela Orús MP, Osma Chacharro S, Ortiz García-Diego N, Chirivella Ramón MT. Uso de la terapia de presión negativa en el tratamiento de las úlceras de pie diabético. Angiologia. 2015;67(5):427-30.

6. Dzieciuchowicz L, Espinosa G, Grochowicz L. El sistema de cierre asistido al vacío en el tratamiento del pie diabético avanzado. Cir Esp. 2009;86(4):213-8.

7. Xia C, Yu A, Qi B, Zhou M, Li Z, Wang W. Analysis of blood flow and local expression of angiogenesis-associated growth factors in infected wounds treated with negative pressure wound therapy. Mol Med Rep. 2014;9:1749-54.

8. Yao M, Fabbi M, Hayashi H, Park N, Attala K, Gu G, et al. A retrospective cohort study evaluating efficacy in high-risk patients with chronic lower extremity ulcers treated with negative pressure wound therapy. Int Wound J. 2014;11(5):483-8. 
9. Monsen C, Acosta S, Mani K, Wann-Hansson C. A randomised study of NPWT closure versus alginate dressings in peri-vascular groin infections: quality of life, pain and cost. J Wound Care. 2015;24(6):25260.

10. De Caridi G, Massara M, Greco M, Pipitò N, Spinelli F, Grande R, et al. VAC therapy to promote wound healing after surgical revascularisation for critical lower limb ischaemia. Int Wound J. 2016;13(3):33642.

11. Nain PS, Uppal SK, Garg R, Bajaj K, Garg S. Role of Negative Pressure Wound Therapy in Healing of Diabetic Foot Ulcers. J Surg Tech Case Rep. 2011;3(1):17-22.

12. Lavery LA, Barnes SA, Keith MS, Seaman JW, Armstrong DG. Prediction of healing for postoperative diabetic foot wounds based on early wound area progression. Diabetes Care. 2008;31(1):26-9.

13. Akbari A, Moodi H, Ghiasi F, Sagheb H, Rashidi H. Effects of vacuum-compression therapy on healing of diabetic foot ulcers: Randomized controlled trial. J Rehabil Res Dev [Internet]. 2007;44(5):631-6. Disponible en: http://www.ncbi.nlm.nih.gov/entrez/query.fcgi?cmd=Retrieve\&db=PubMed\&dopt=Citation\&list uids $=17943674$.

14. Lone AM, Zaroo MI, Laway BA, Pala NA, Bashir SA, Rasool A. Vacuum-assisted closure versus conventional dressings in the management of diabetic foot ulcers: A prospective case-control study. Diabet Foot Ankle. 2014;5:23345.

15. Eginton MT, Brown KR, Seabrook GR, Towne JB, Cambria RA. A Prospective Randomized Evaluation of Negative-pressure Wound Dressings for Diabetic Foot Wounds. Ann Vasc Surg. 2003;17(6):645-9.

16. Blume P, Walters J, Payne W, Ayala J, Lantis J. Comparison of Negative Pressure Wound Therapy Using Vacuum-Assisted Closure With Advanced Moist Wound Therapy in the Treatment of Diabetic Foot Ulcers: A multicenter randomized controlled trial. Diabetes Care. 2008;31(4):631-6.

17. Lavery, L A. Fontaine, J. Thakral, G. Kim, P J. Bhavan, K. Davis K. Randomized Clinical Trial to Compare Negative- Pressure Wound Therapy Approaches with Low and High Pressure, Silicone- Coated Dressing, and Polyurethane Foam Dressing. Plast Reconstr Surg. 2014;133(3):722-6.

18. Sepúlveda G, Espíndola M, Maureira M, Sepúlveda E, Fernández JI, Oliva C, et al. Curación asistida por presión negativa comparada con curación convencional en el tratamiento del pie diabético amputado. Ensayo clínico aleatorio. Cir Esp. 2009;86(3):171-7.

19. Etoz A, Ozgenel Y, Ozcan M. The use of negative pressure wound therapy on diabetic foot ulcers: a preliminary controlled trial. Wounds. 2004;16(8):264-9.

20. Karatepe O, Eken I, Acet E, Unal O, Mert M, Koc B, et al. Vacuum assisted closure improves the quality of life in patients with diabetic foot. Acta Chir Belg. 2016;111(5):298-302.

21. Yang S-L, Han R, Liu Y, Hu L-Y, Li X-L, Zhu L-Y. Negative pressure wound therapy is associated with up-regulation of bFGF and ERK1/2 in human diabetic foot wounds. Wound Repair Regen [Internet]. 2014;22(4):548-54. Disponible en: http://www.ncbi.nlm.nih.gov/pubmed/24809625.

22. Apelqvist J, Armstrong DG, Lavery LA, Boulton AJM. Resource utilization and economic costs of care based on a randomized trial of vacuum-assisted closure therapy in the treatment of diabetic foot wounds. Am J Surg. junio de 2008;195(6):782-8.

23. Seidel D, Mathes T, Lefering R, Storck M, Lawall H, Neugebauer EAM. Negative pressure wound therapy versus standard wound care in chronic diabetic foot wounds: study protocol for a randomized controlled trial. Trials. 2014;15:334.

24. Braakenburg A, Obdeijn MC, Feitz R, van Rooij I a LM, van Griethuysen AJ, Klinkenbijl JHG. The clinical efficacy and cost effectiveness of the vacuum-assisted closure technique in the management of acute and chronic wounds: a randomized controlled trial. Plast Reconstr Surg. 2006;118(2):390-7.

25. Whitehead SJ, Forest-Bendien VL, Richard JL, Halimi S, Van GH, Trueman P. Economic evaluation of Vacuum Assisted Closure ${ }^{(\mathrm{R})}$ Therapy for the treatment of diabetic foot ulcers in France. Int Wound J. 2011;8:22-32.

26. Trujillo-Martín M, García-Pérez L, Serrano-Aguilar P. Efectividad, seguridad y coste-efectividad de la terapia por presión negativa tópica para el tratamiento de las heridas crónicas: una revisión sistemática. Med Clin (Barc). 2011;137(7):321-8.

27. Driver VR, Blume PA. Evaluation of wound care and health-care use costs in patients with diabetic foot ulcers treated with negative pressure wound therapy versus advanced moist wound therapy. J Am Podiatr Med Assoc. 2014;104(2):147-53.

28. Trueman P. Cost-effectiveness considerations for home health V.A.C. Therapy in the United States of America and its potential international application. Int Wound J. 2008;5(2):23-6.

29. Hutton DW, Sheehan P. Comparative effectiveness of the SNaP ${ }^{\mathrm{TM}}$ Wound Care System. Int Wound J. 2011;8(2):196-205.

30. Lerman B, Oldenbrook L, Ryu J, Fong KD, Schubart PJ. The SNaP Wound Care System: a case series using a novel ultraportable negative pressure wound therapy device for the treatment of diabetic lower extremity wounds. J Diabetes Sci Technol. 2010;4(4):825-30. 
31. Atema JJ, Gans SL, Boermeester MA. Systematic review and meta-analysis of the open abdomen and temporary abdominal closure techniques in non-trauma patients. World J Surg. 2015;39(4):912-25.

32. Bruhin A, Ferreira F, Chariker M, Smith J, Runkel N. Systematic review and evidence based recommendations for the use of Negative Pressure Wound Therapy in the open abdomen. Int J Surg [Internet]. 2014;12(10):1105-14. Disponible en: http://www.journal-surgery.net/article/S1743-9191(14)00881-4/ pdf

33. Hyldig N, Birke-Sorensen H, Kruse M, Vinter C, Joergensen JS, Sorensen JA, et al. Meta-analysis of negative-pressure wound therapy for closed surgical incisions. Br J Surg. 2016;103(5):477-86.

34. Kantak NA, Mistry R, Halvorson EG. A review of negative-pressure wound therapy in the management of burn wounds. Burns [Internet]. 2016; Disponible en: http://www.burnsjournal.com/article/S03054179(16)30182-6/pdf

35. Schlatterer DR, Hirschfeld AG, Webb LX. Negative Pressure Wound Therapy in Grade IIIB Tibial Fractures: Fewer Infections and Fewer Flap Procedures? Clin Orthop Relat Res. 2015;473(5):1802-11.

36. Shrestha BM. Systematic review of the negative pressure wound therapy in kidney transplant recipients. World J Transplant. 2016;6(4):767.

37. Guffanti A. Negative Pressure Wound Therapy in the Treatment of Diabetic Foot Ulcers: A Systematic Review of the Literature. J Wound, Ostomy Cont Nurs. 201416];41(3):233-7.

38. Zhang J, Hu Z-C, Chen D, Guo D, Zhu J-Y, Tang B. Effectiveness and safety of negative pressure wound therapy for diabetic foot ulcers: a meta-analysis. Plast Reconstr Surg. 2014;134:141-51. 